\title{
Consta-Abelian codes over Galois rings in the DFT domain
}

\author{
Kiran. T, and B. Sundar Rajan \\ ECE Dept., Indian Institute of Science, Bangalore 560 012, India \\ \{kirant@protocol., bsrajan@\}ece.iisc.ernet.in
}

\begin{abstract}
Using Twisted-DFT, we characterize Consta-Abelian codes over Galois rings that are closed under two kinds of monomials.
\end{abstract}

\section{Introduction and Preliminaries}

For a prime $p$, Galois ring $R_{p^{a}, l}=Z_{p^{a}}[x] / \phi(x)$, where $\phi(x)$ is a monic irreducible polynomial of degree $l$ over $Z_{p}[x][1]$. If $G=$ $C_{r-1} \otimes \cdots \otimes C_{0}$, is an Abelian group of order $n=\prod_{\lambda=0}^{r-1} m_{\lambda}$, the twisted group ring $R_{p^{a}, l}^{\psi} G$ [2] is defined by imposing in the $R_{p^{a}, l}$-module $R_{p^{a}, l} G$ the following multiplication on each of the generators $g_{\left(m_{k}\right)}$ of the cyclic subgroup $C_{k}: g_{\left(m_{k}\right)}^{i} \cdot g_{\left(m_{k}\right)}^{j}=$ $\psi_{k}\left(g_{\left(m_{k}\right)}^{i}, g_{\left(m_{k}\right)}^{j}\right) g_{\left(m_{k}\right)}^{i+j}$, where $g_{\left(m_{k}\right)}^{i+j} \in G$ and

$$
\psi_{k}\left(g_{\left(m_{k}\right)}^{i}, g_{\left(m_{k}\right)}^{j}\right)=\left\{\begin{array}{cl}
1 & \text { if } i+j<m_{k} \\
\beta_{k} & \text { if } i+j \geq m_{k}
\end{array}\right.
$$

where $\beta_{k}$, an element of order $r_{k}$ belongs to the cyclic subgroup of size $p^{l}-1$ in $R_{p^{a}, l}^{*}$. For $n$ permutations $\pi_{0}, \ldots, \pi_{n-1}$ of $R_{p a, l}$ and a permutation $\tau$ of $I_{n}=\{0,1, \cdots, n-1\}$ a code $\mathcal{C}$ over $R_{p^{a}, l}$ is said to be $\pi \tau$-invariant if $\pi \tau(\vec{c})=$ $\left(\pi_{0}\left(c_{\tau(0)}\right), \pi_{1}\left(c_{\tau(1)}\right), \ldots, \pi_{n-1}\left(c_{\tau(n-1)}\right)\right) \in \mathcal{C}$ for all $\vec{c}=$ $\left(c_{0}, c_{1}, \ldots, c_{n-1}\right) \in \mathcal{C}$. Consta-Abelian codes are ideals in the twisted group ring $R_{p^{a}, 1}^{\psi} G$. For $\bar{\beta}=\left(\beta_{r-1}, \ldots, \beta_{0}\right)$ these are also called $\bar{\beta}$-constacyclic codes. For every codeword $\vec{c}=\left(c_{0}, \ldots, c_{n-1}\right)$ in the $\bar{\beta}$-constacyclic code, the monomialpermuted vector $\pi \tau(\vec{c})=\left(\pi_{0}\left(c_{0 \oplus j}\right), \ldots, \pi_{n-1}\left(c_{(n-1) \oplus j}\right)\right)$ also belongs to the code for all values of $j$, where $\pi_{i}\left(c_{i \oplus j}\right)=$ $\left(\prod_{\lambda=0}^{r-1} \beta_{\lambda}^{k_{\lambda}}\right) c_{i \oplus j}$ and $k_{\lambda}$ is such that $i_{\lambda}+j_{\lambda}=m_{\lambda} k_{\lambda}+\left(i_{\lambda} \oplus j_{\lambda}\right)$ where $i \oplus j$ denotes the mixed-radix addition with $m_{\lambda}$ as radixes [3]. Let $\zeta_{\lambda}$ be a primitive $m_{\lambda} r_{\lambda}$-th root of unity in the cyclic group of order $p^{l m}-1$ in the extension ring $R_{p^{a}, l m}$ and $\gamma_{\lambda}=\zeta_{\lambda}^{h_{\lambda}}, \alpha_{\lambda}=\zeta_{\lambda}^{r_{\lambda}}$ be $m_{\lambda}$-th root of $\beta_{\lambda}$ and $m_{\lambda}$-th root of unity respectively for all $\lambda=0,1, \ldots, r-1$. The TDFT vector $\vec{A}=\left(A_{0}, \ldots, A_{n-1}\right) \in R_{p^{a}, l m}^{n}$ of $\vec{a}=\left(a_{0}, \ldots, a_{n-1}\right) \in$ $R_{p^{a}, l}^{n}$ is defined as $A_{j}=\sum_{i=0}^{n-1}\left(\prod_{\lambda=0}^{r-1} \gamma_{\lambda}^{i_{\lambda}} \alpha_{\lambda}^{i_{\lambda} j_{\lambda}}\right) a_{i}$ where

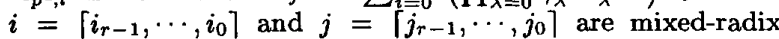
representations of $i, j$. The TDFT satisfies all the properties mentioned in [4] for a constacyclic DFT. Let $\sigma_{0}$ be the Frobenius automorphism of $R_{p^{a}, l m}$ then $\sigma=\sigma_{0}^{l}$ is an automorphism of $R_{p^{a}, l m}$ that fixes $R_{p^{a}, l} \subseteq R_{p^{a}, l m}$. Moreover for $j \in I_{n}, \sigma^{k}\left(A_{j}\right)=A_{i}$, where $i=\left\lceil i_{r-1}, i_{r-2}, \ldots, i_{0}\right\rceil$ with $i_{\lambda}=\left(\frac{\left(q^{k}-1\right) h_{\lambda}}{r_{\lambda}}+q^{k} j_{\lambda}\right)$ modulo $m_{\lambda}$ for all $\lambda=0,1, \ldots, r-1$ (Conjugate symmetry property). Let $\phi_{\lambda}^{k}$ denote a mapping from $I_{m_{\lambda}}$ to $I_{m_{\lambda}}$ which maps $j_{\lambda}$ to $\left(\frac{\left(q^{k}-1\right) h_{\lambda}}{r_{\lambda}}+q^{k} j_{\lambda}\right)$. Let $\Phi^{k}$ denote the mapping $i=\left\lceil i_{r-1}, i_{r-2}, \ldots, i_{0}\right\rceil$ to $\Phi^{k}(i)=$ $\left\lceil\phi_{r-1}^{k}\left(i_{r-1}\right), \phi_{r-2}^{k}\left(i_{r-2}\right), \ldots, \phi_{0}^{k}\left(i_{0}\right)\right\rceil$. For every $j \in I_{n}$, the set $\widehat{\lceil j\rceil}=\left\{\Phi^{1}(j), \Phi^{2}(j), \ldots, \Phi^{e_{j}-1}(j)\right\}$ where $e_{j}$ is the smallest integer such that $\Phi^{e_{j}}(j)=j$, is called the cyclotomic coset

${ }^{1}$ This work was partly supported by CSIR, India, through Research Grant (22(0298)/99/EMR-II) to B.S.Rajan. modulo $n$ containing $\lceil j\rceil$. A linear code $\mathcal{C}$ over $R_{p^{a}, l}$ is constaAbelian iff it satisfies the conjugate-symmetry property and the set $\mathrm{C}_{\mathrm{j}}=\left\{A_{j} \mid \vec{a} \in \mathcal{C}\right\}=p^{\eta_{j}} R_{p^{a}, l e_{j}}$, an ideal of the subring $R_{p^{a}, l_{j}}$ where $0 \leq \eta_{j} \leq a$.

In this paper, we study $\bar{\beta}$-constacyclic codes which are also invariant under the monomial-permutations $\pi^{(b)} U_{b}$ and $\pi^{<s>} Q_{n}$, where: (i) $U_{b}$ is defined by, $\lceil i\rceil=\left\lceil i_{r-1}, \ldots, i_{0}\right\rceil \rightarrow$ $\left\lceil b_{r-1} i_{r-1}, \ldots, b_{0} i_{0}\right\rceil$ for $b=\left\lceil b_{r-1}, \ldots, b_{0}\right\rceil \in I_{n}$ such that $\operatorname{gcd}\left(b_{\lambda}, m_{\lambda}\right)=1$ and $r_{\lambda} \mid\left(b_{\lambda}^{-1}-1\right)$ for all $\lambda$. The associated permutation $\pi^{(b)}=\left(\pi_{0}^{(b)}, \ldots, \pi_{r-1}^{(b)}\right)$ is given by $\pi_{i}^{(b)}\left(a_{i}\right)=\prod_{k=0}^{r-1} \psi_{k}\left(g_{m_{k}}^{b_{k}^{-1} i_{k}} . a_{i}\right)$ and (ii) for $n_{s}=\prod_{\lambda=0}^{s-1} m_{\lambda}$ $Q_{n_{s}}$ takes $i \rightarrow\left(i+n_{s}\right)$ modulo $n$ and the associated permutation $\pi^{\langle s\rangle}=\left(\pi_{0}^{\langle s\rangle}, \ldots, \pi_{r-1}^{\langle s\rangle}\right)$ is given by $\pi_{i}^{\langle s\rangle}\left(a_{i}\right)=a_{i}$ for $0 \leq i<n_{s+1}-n_{s}$ and for $n_{s+1}-n_{s} \leq i<n$, $\pi_{i}^{<s>}\left(a_{i}\right)=\prod_{k=s}^{r-1} \psi_{k}\left(g_{m_{k}}^{i_{k}+\delta_{k}^{(s)}} a_{i}\right)$, where $\delta_{k}^{(s)}$ denotes the "carry value" in the $k$-th radix-component due to addition $\left\lceil i+n_{s}\right\rceil$.

\section{TDFT DOMAIN CHARACTERIZATION}

Definition 1 (i) For any $j \in I_{n}$ such that $\lceil j\rceil=\left[0,0, \ldots, 0, j_{\text {ptf }}\right.$ $\left.0, j_{\mu-1}, \ldots, j_{0}\right\rceil$ and $\mu \geq h>s \geq 0$, let the set $J^{(h, s)}(j)$ be $\left\{\left[0, \cdots, 0, j_{\mu}, \ldots, j_{h}, x_{h-1}, x_{h-2}, \ldots, x_{s}, j_{s-1}, \ldots, j_{0}\right\rceil\right\}$ for all $x_{\lambda} \in I_{m_{\lambda}} ; \lambda=h-1, h-2, \ldots, s$. (ii) For every $\lambda=0,1, \ldots, r-1$, let $\phi_{\lambda}^{k, b_{\lambda}}$ denote the mapping from $I_{m_{\lambda}}$ to $I_{m_{\lambda}}$ that maps $j_{\lambda}$ to $\left(\frac{\left(b_{\lambda}^{-k}-1\right) h_{\lambda}}{r_{\lambda}}+b_{\lambda}^{-k} j_{\lambda}\right)$ and let $\Phi^{k, b}$ be the map which maps $\lceil j\rceil$ to $\Phi^{k, b}(\lceil j\rceil)=\left\lceil\phi_{r-1}^{k, b_{r-1}}\left(j_{r-1}\right), \phi_{r-2}^{k, b_{r-2}}\left(j_{r-2}\right), \ldots, \phi_{0}^{k, b_{0}}\left(j_{0}\right)\right\rceil$.

Theorem 1 A length $n=m_{r-1} m_{r-2} \cdots m_{0} \bar{\beta}$-constacyclic code with $\mathbf{C}_{\mathbf{j}}=p^{\eta_{j}} R_{p^{a}, l e_{j}}$ for any $j \in I_{n}$ is (i) $\pi^{(b)} U_{b}$ invariant iff $\mathbf{C}_{\mathbf{i}}=p^{\eta_{j}} R_{p^{a}, l_{i}}$ when some element of $\widehat{\lceil i\rceil}$ is of the form $\Phi^{k, b}(\lceil j\rceil)$ for some $k$. (ii). $\pi^{<s>} Q_{n_{s}}$-invariant iff $\mathbf{C}_{\mathbf{i}}=p^{\eta_{j}} R_{p^{a}, l_{i}}$ when $j \geq n_{s+1}$ and some element of $\widehat{\lceil i\rceil}$ belongs to $J^{(r-1, s)}(j)$.

For the special case of $\beta_{\lambda}=1$ for all $\lambda$, Theorem 1 characterizes (i) Quasicyclic-Abelian codes and (ii) $U_{b}$-invariant Abelian codes. Given the TDFT characterization of a $\bar{\beta}$ constacyclic code, we obtain the characterization of its dual code (w.r.t normal inner product).

\section{REFERENCES}

[1] B.R.McDonald, "Finite Rings with Identity," Marcel Dekker Inc., 1974, New York.

[2] G.Hughes, "Structure theorems for Group Ring Codes with an application to Self-Dual Codes," Des. Codes. Cryptogr., vol. 24, pp. 5-14, Sept-2001.

[3] B.Sundar Rajan and M.U.Siddiqi, "A Generalized DFT for Abelian Codes over $Z_{m}$," IEEE Trans. Info. Theory, vol. 40, no. 6, pp. 2082-2090, Nov-1994.

[4] Serdar Boztas, "Constacyclic Codes and Constacyclic DFTs." Proc. of ISIT-1998, pp. 235, Cambridge, MA, USA, 